Article

\title{
Improving Diagnosis of Cervical Pre-Cancer: Combination of PCA and SVM Applied on Fluorescence Lifetime Images
}

\author{
Gyana Ranjan Sahoo ${ }^{1} \mathbb{D}$, Pankaj Singh ${ }^{1}$, Kiran Pandey ${ }^{2}$, Chayanika Kala ${ }^{3}$ and \\ Asima Pradhan 1,4,*(D) \\ 1 Department of Physics, IIT Kanpur, Kanpur 208016, India; gyana@iitk.ac.in (G.R.S.); singhp@iitk.ac.in (P.S.) \\ 2 Department of Obstetrics \& Gynaecology, GSVM Medical College Kanpur, Kanpur 208002, India; \\ dr.kiranpandey@gmail.com \\ 3 Department of Pathology, LPS Institute of Cadiology, Kanpur 208019, India; drchayanikakala@yahoo.com \\ 4 Centre for Lasers and Photonics, IIT Kanpur, Kanpur 208016, India \\ * Correspondence: asima@iitk.ac.in; Tel.: +91-0512-259-7971
}

Received: 15 October 2018; Accepted: 26 November 2018; Published: 10 November 2018

\begin{abstract}
We report a significant improvement in the diagnosis of cervical cancer through a combined application of principal component analysis (PCA) and support vector machine (SVM) on the average fluorescence decay profile of Fluorescence Lifetime Images (FLI) of epithelial hyperplasia (EH) and CIN-I cervical tissue samples, obtained ex-vivo. The fast and slow components of double exponential fitted fluorescence lifetimes were found to be higher for $\mathrm{EH}$ compared to the lifetimes of CIN-I samples. Application of PCA to the average time-resolved fluorescence decay profiles showed that the 2nd PC, in combination with 1st PC, enhanced the discrimination between EH and CIN-I tissues. Fluorescence lifetime and PC scores were then classified separately by using SVM support vector machine to identify the two. On applying SVM to a combination of fluorescence lifetime and PC scores, diagnostic capability improved significantly.
\end{abstract}

Keywords: fluorescence lifetime; PCA; PC scores; SVM

\section{Introduction}

Since the early 1980s, steady-state fluorescence spectroscopy has been extensively studied by several groups for detection of cancer [1-9]. Fluorescence spectra have been utilizused by several groups to detect cervical cancer $[6,9,10]$. Ramanujan et al. have efficiently combined reflectance with fluorescence spectroscopy for in-vivo cervical pre-cancer detection $[9,11]$, which has resulted in a fiber based probe for clinical studies. However, one of the limitations of fluorescence spectroscopy from biological tissue is the highly overlapping character of contributing fluorophores making it difficult to understand disease development through the changes in fluorophores. In addition, it is difficult to interpret or quantify the difference in spectra due to quenching by other molecules, aggregation, or energy transfer, because fluorescence intensity depends on fluorophore concentration and illumination intensity. On the other hand, fluorescence lifetime $(\tau)$ is independent of fluorophore concentration and illumination intensity but depends only on the intrinsic characteristics. It is also sensitive to the local environment such as viscosity, $\mathrm{pH}$, refractive index as well as interactions with other molecules [12-14]. During the progress of cancer, the environment of the contributing fluorophores changes and this can be captured by its lifetime as it is more sensitive to environment as compared to steady-state fluorescence $[15,16]$. Hence, the temporal information adds an extra dimension which can be used to distinguish different fluorophores with overlapping spectra 
but exhibiting different lifetimes. The photo-physical properties of the intrinsic bio-molecules and bio-structures have been considered as a possible parameter that may be related to the morphofunctional state of a biological tissue. Due to this advantage, time-resolved fluorescence technique has been used to study the structure and dynamics [17-21] of biological molecules and has been applied in tissue diagnosis [21,22], specifically for cervical cancer detection. [22,23].

Tissue undergoes changes during the development of cancer. Morphological changes mostly contribute to changes in the scattering properties, while biochemical changes occur at a cellular level from bio-molecules which are reflected in the absorption properties of the tissue [24,25]. In addition, tissue undergoes changes in concentration of fluorophores e.g., decrease in concentration of flavin adenine dinucleotide (FAD) and increase in concentration of nicotinamide adenine dinucleotide (NADH) which are well documented [26,27]. Optical techniques may be more sensitive to the changes occurring in the preliminary stages of cancer than the existing conventional techniques [25,28].

Further, application of PCA on fluorescence lifetime images improves the capabilities of the lifetime, since the principal components capture both absorption and scattering effects [29]. PCA is a well known technique used for dimensionality reduction of data [30]. It has been used by several groups for cancer diagnosis by feature extraction [26,29,31-33]. Recently, different organs have been differentiated using this technique, in which changes in anatomical behavior is reflected in its time corelated fluorescence signal [34-36]. Statistical algorithms play an important role in enhancing the discrimination. The physiological changes in each organ lead to different fluorescence decay times. These are enhanced through different principal components, which provide structural information of organs. In another study, our group has validated with the phantom studies, how the PCs capture the absorption and scattering information and thus clearly demarcate the precancerous regions from the cancerous sites [29].

For better classification of cervical data, comparative evaluation of several classifier techniques using same set of data is necessary. Among all the available classifiers, SVM is robust and performs better than other classification techniques. SVM is a machine learning algorithm used for classification of data, function approximation, etc., due to its generalization ability, it has been successfully applied in many cases [37-43]. SVM works by minimizing the upper bound of error through maximizing the margin between separating hyperplane and data set. It has the advantage of choosing the model automatically such that both the optimal number and location of basis functions are determined during training. SVM is also suitable for small samples and some inherently non-linear problems. There are several kernel functions available for SVM, both for linear and non-linear classification, and its performance largely depends on the selection of the kernel function [44,45]. Rarely is one index test sufficient for diagnosis of a particular condition, and so diagnostics involving multiple tests are often used. We have hence, utilizused the advantage of SVM on fluorescence lifetime and PC scores for classification.

Here, we report the discrimination of EH and CIN-I through their fluorescence lifetime. The average fluorescence decay profiles were fitted to a double exponential. A principal component analysis was applied to the time-resolved fluorescence decay and PC scores were calculated for each sample. Fluorescence lifetime and PC scores were used for classification of EH and CIN-I samples by the application of a machine learning algorithm (SVM). We explain how the combination of SVM results of fluorescence lifetime and PCA were used to improve the diagnostic efficacy.

\section{Results}

\subsection{Fluorescence Lifetime}

Here, we have used $375 \mathrm{~nm}$ wavelength pulsed laser for excitation, with the resulting fluorescence dominantly from NADH and FAD [28]. Fluorescence intensity images at a certain time delay are shown in Figure 1a,d for EH and CIN-II, respectively. The corresponding FLI are shown in Figure 1b,e for fast component $\left(\tau_{1}\right)$ and Figure $1 \mathrm{c}, \mathrm{f}$ for slow component $\left(\tau_{2}\right)$. Both the fast and slow components of 
FLI for EH are higher than those of CIN-I tissues. However, fluorescence lifetime at few pixels are higher due to bad fitting at those pixels. Fluorescence decay was found to be faster for CIN-I compared to EH samples, as indicated in Figure 2a. Scatter plot of fast versus slow components of fluorescence lifetimes of fitted average fluorescence decay of all the samples for EH and CIN-I tissues is shown in Figure $2 \mathrm{~b}$. The fast and slow components of average fluorescence lifetime also show higher values for most of the EH as compared to CIN-I tissues as illustrated in Figure $2 b$.

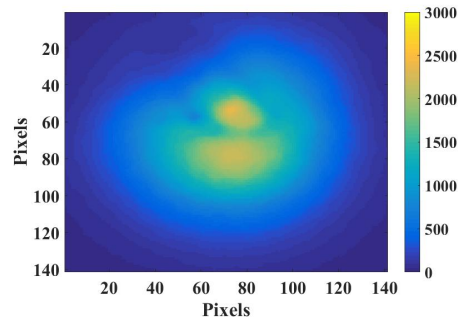

(a)

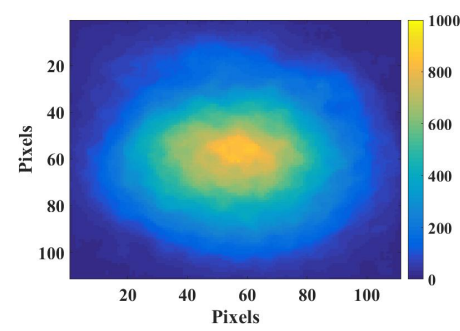

(d)

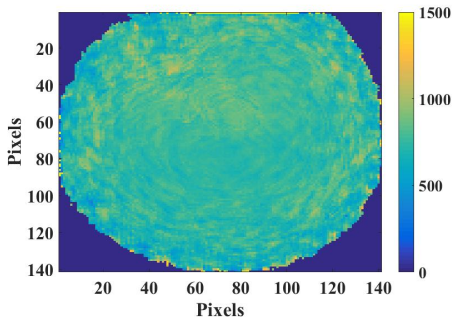

(b)

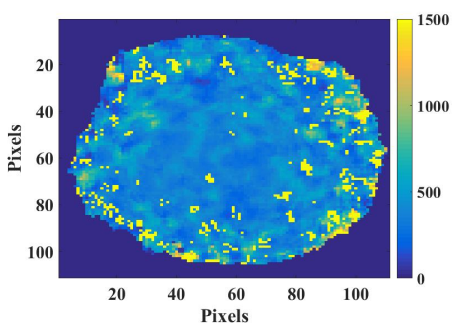

(e)

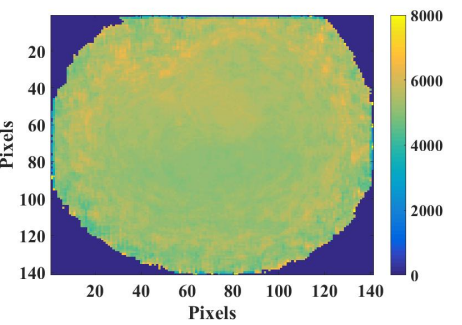

(c)

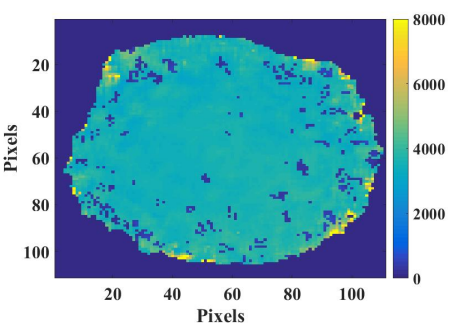

(f)

Figure 1. (a,d) The fluorescence intensity image at a certain time delay; $(\mathbf{b}, \mathbf{c}, \mathbf{e}, \mathbf{f})$ corresponding fluorescence lifetime images of fast and slow components for $\mathrm{EH}$ and CIN-II, respectively.

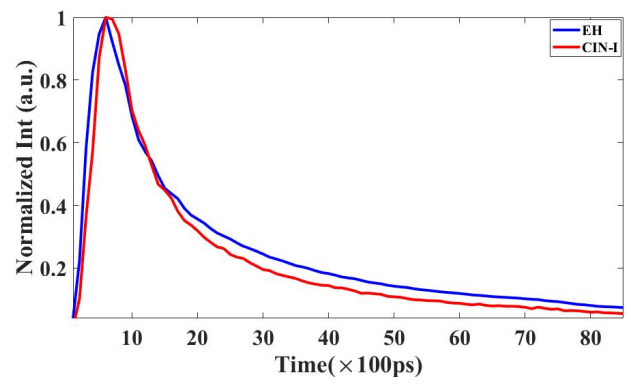

(a)

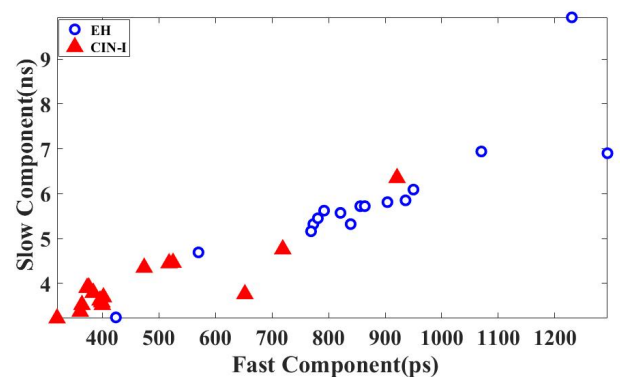

(b)

Figure 2. (a) Average fluorescence decay profiles and (b) Scatter plot of fast versus slow components of fluorescence lifetimes for $\mathrm{EH}$ and CIN-I sample.

\subsection{Principal Component Analysis}

PCA has been performed on average time-resolved fluorescence decay profiles following the method mentioned in Section 4.3.2. First three eigenvalues are considered here as they capture more than $99.5 \%$ of the total variance of the fluorescence decay. This can be seen in the eigenvalue and variance plots shown in Figure 3a. PC scores represent the projection of data on the principal components and hence carry useful information, which can be used for classification purposes. Scatter plots between 1st and 2nd PC scores, 2nd and 3rd PC scores and 1st and 3rd PC scores are shown in Figure $3 b-d$, respectively. It can be seen from Figure $3 b$ that the EH and CIN-I tissue samples can be distinguished clearly from the scatter plot between 1st and 2nd PC scores, but the distinction becomes clearer from the scatter plot between 2nd and 3rd PC scores as seen in Figure 3c. From Figure 3d, it can be seen that the scatter plot between 1st and 3rd PC scores for both EH and CIN-I samples 
are highly overlapping and hence, the 3rd PC does not carry useful information, which can be used for classification.

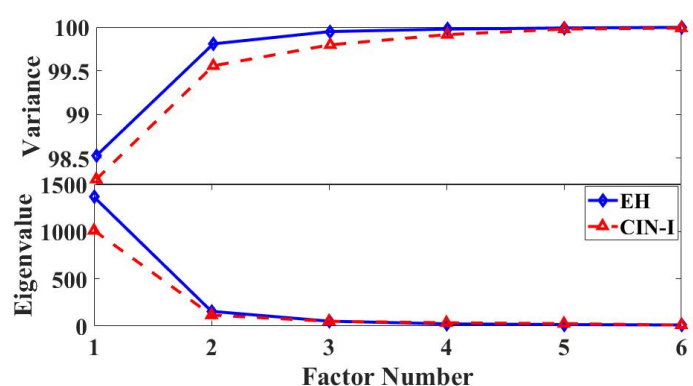

(a)

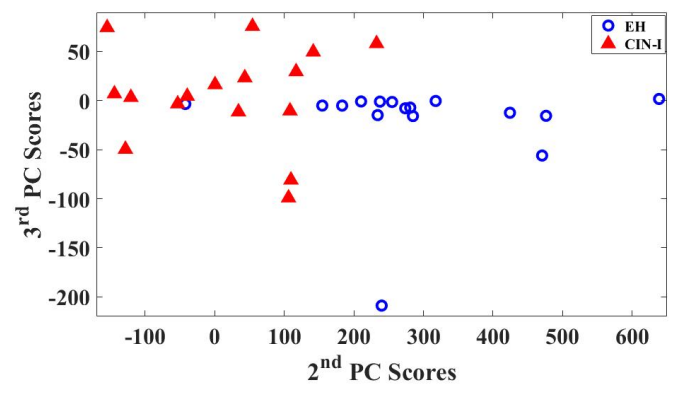

(c)

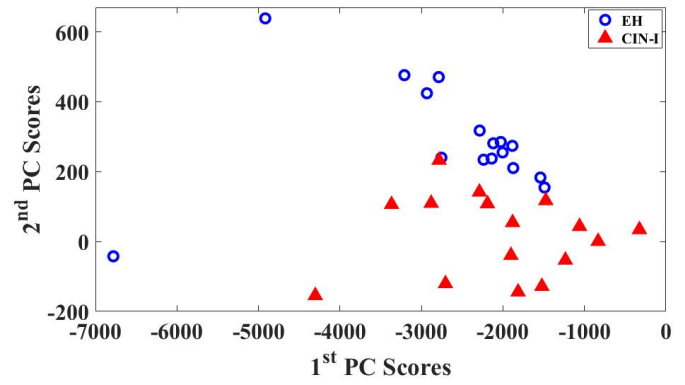

(b)

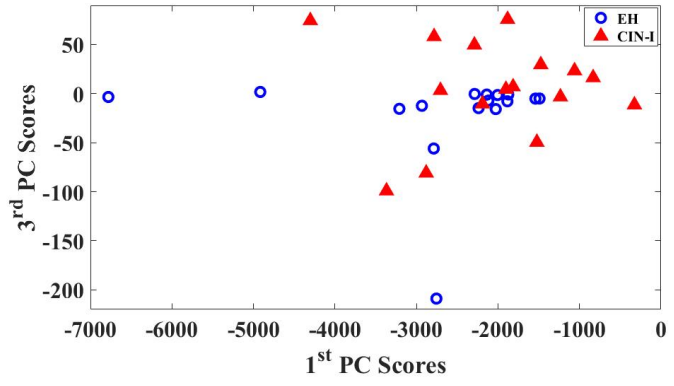

(d)

Figure 3. (a) Eigenvalue and variance plots of first six eigenvalues, (b) Scatter plot of 1st PC versus 2nd PC, (c) 2nd PC versus 3rd PC and (d) 1st PC versus 3rd PC scores for EH and CIN-I cervical tissue.

\subsection{Support Vector Machine}

The SVM is used for classification of the processed data. The important features of SVM are mapping linear inseparable data into high-dimensional space by non-linear kernel function and linearly distinguishing the data in high-dimensional space. SVM is especially suitable for small samples and some inherently non-linear problems. The data points that lies close to the decision surface receives the maximum weightage and the points far away from the margin receives zero weightage. These data points close to the decision boundary are called support vectors. The margin of the classifier is determined by the distance from the decision surface to the support vectors. In SVM, the holdout method of cross- validation was employed to randomly divide the data set into two parts: a training set consisting of $16 \mathrm{EH}$ and $16 \mathrm{CIN}-\mathrm{I}$ samples and validation set includes $9 \mathrm{EH}$ and $4 \mathrm{CIN}-\mathrm{I}$ and $2 \mathrm{CIN}-\mathrm{II}$ samples. Here, the input data matrices for SVM classifier were dimension reduced by application of PCA. The first two PCs are considered as they occupy more than $99 \%$ of total information. To obtain the optimal parameter of the classifier, a grid search technique with tenfold cross-validation was employed. Given a constant sample size, one approach to improve the classification accuracy may be to incorporate non-linear techniques such as non-linear kernels in SVM analysis. Different types of linear and non-linear kernel functions were tried, out of which "radial basis function (RBF)" Kernel was found to classify better than others. Figure $4 a, c$ show the plot of training data results between fast and slow components of lifetime and 1st and 2nd PC scores respectively, which are separated by well defined $\mathrm{EH}$ and CIN-I regions. Corresponding validation data results are shown in Figure $4 \mathrm{~b}, \mathrm{~d}$. In both the cases, the two CIN-I samples are lying outside the CIN-I regions, while all the normal samples are clearly distinguished.

Here we have used two analysis techniques for detection of cervical cancer. To improve the diagnosis, we combined the results obtained from both the techniques. To improve the sensitivity of the test it is considered positive if one result is positive. The final sensitivity (Sn) and specificity (Sp) of the 
combined tests (A and B) used in this work is, $S n_{A B}=S n_{A}+S n_{B}-S n_{A} \times S n_{B}$ ans $S p_{A B}=S p_{A} \times S p_{B}$. The calculated combined specificity and sensitivity are $87 \%$ and $100 \%$ respectively.

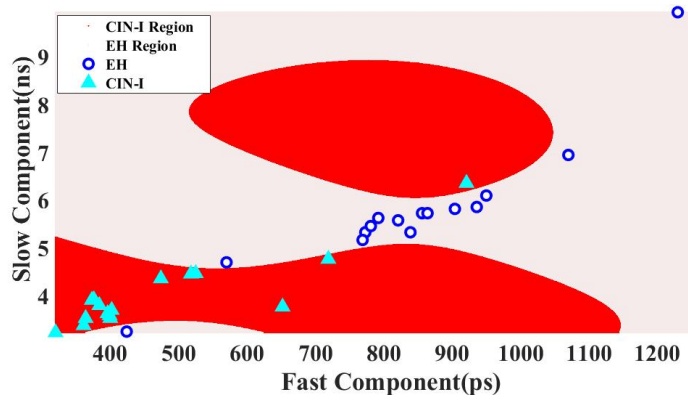

(a)

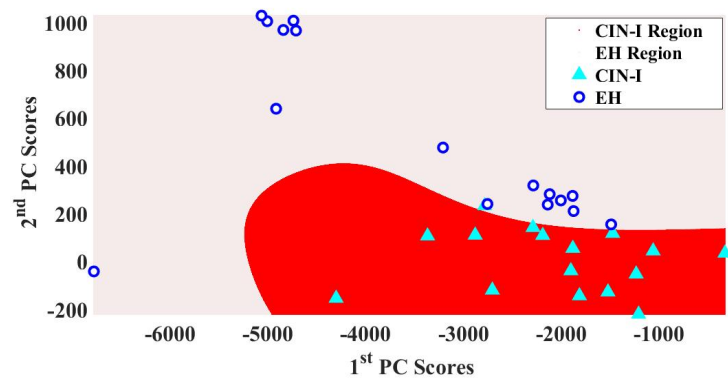

(c)

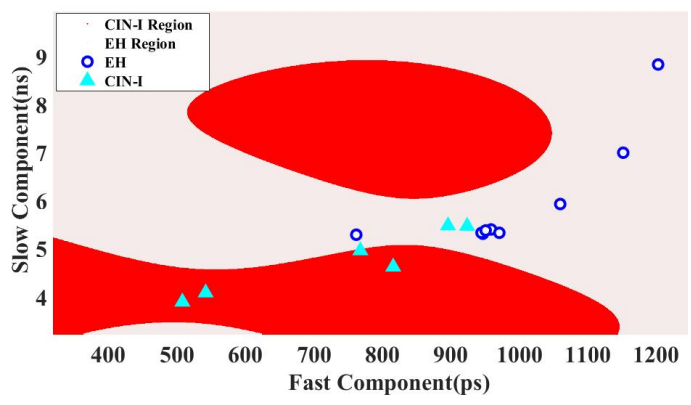

(b)

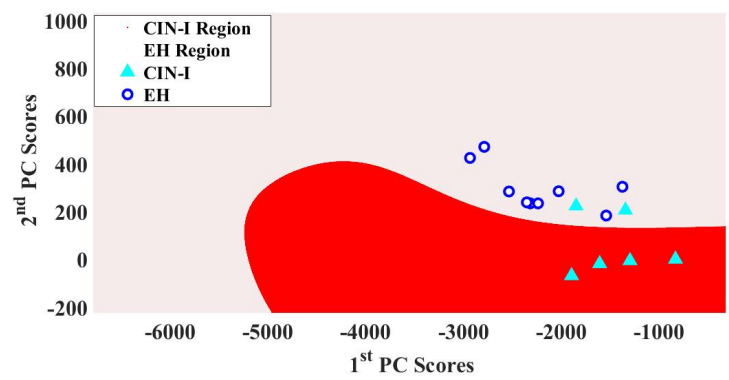

(d)

Figure 4. The RBF-SVM based classification of EH and CIN-I cervical tissues for fast component versus slow component (a) training and (b) validation; 1st PC versus 2nd PC (c) training and (d) validation data sets.

\section{Discussion}

\subsection{Fluorescence Lifetime}

Both the fast and slow components of FLI shown in Figure $1 \mathrm{~b}$,e and c,f respectively are found to be higher for $\mathrm{EH}$ than those of CIN-I tissue. It may be noted that the low SNR at the edges of the images create error in fitting, giving rise to higher values of lifetime. The average fluorescence decay profile, on the other hand, shows a better SNR and hence its double exponential fitting is more reliable. On comparing the lifetimes obtained from the images and the average fluorescence decay profiles, they are found to match well where the SNR are high. On comparing the fluorescence lifetime for all tissues from both the categories, both the fast and slow components of lifetime are found to be higher for EH than CIN-I, which can be confirmed from Figure 2b. In our earlier results we showed that the fluorescence lifetime for normal tissue is lesser than that of CIN-I tissue [29] but, it is pertinent to note that in this study, we report results of $\mathrm{EH}$ rather than normal tissues. $\mathrm{EH}$ is declared as normal by the histopathologist, but, its epithelial thickness is almost double of a typical normal tissue. The fluorescence signal would then emerge from deeper regions undergoing higher number of scattering events before escaping from the tissue, which would result in an increase in fluorescence lifetime.

\subsection{Principal Component Analysis}

Fluorescence lifetime shows a good discrimination between the EH and CIN-I tissues, but the double exponential fitting is prone to error in case of noisy data and hence cannot be trusted in case of low SNR [46]. This can be confirmed from Figure 1b,c,e,f where lifetime for few pixels show high values due to bad fitting because of low SNR. PCA helps to overcome this limitation. It has been shown that fluorescence lifetime is more sensitive to changes in scattering and is unaffected by absorption. 
Application of PCA to time-resolved fluorescence images has the advantage of capturing both the changes in absorption and scattering of fluorophore environment $[16,29]$. First three eigenvalues are considered here as they capture more than $99.5 \%$ of the total variance of the fluorescence decay as seen in Figure 3a. The eigenvectors corresponding to these three eigenvalues are used to represent the complete data, and reconstruction using these display profiles very close to the original data. From Figure 3d it can be seen that the scatter plot between 1st and 3rd PC scores are highly overlapping, while scatter plots between 1st versus 2nd (Figure 3b) and 2nd versus 3rd (Figure 3c) PC scores shows clear distinction between EH and CIN-I samples. From the above results, one can assume that the 2nd PC plays an important role in discriminating EH and CIN-I tissues as it captures the subtle changes in fluorophore environment i.e., the changes in absorption and scattering, which complies with our earlier results [29].

\subsection{Support Vector Machine}

For PC score classification, first two PCs are considered as they carry more than $99 \%$ of the original information (as seen in Figure 3a) while 3rd PC does not carry any significant information. The overall model results for PC scores using different kernels for training data is shown in Table 1. The performance of a model is generally evaluated in terms of accuracy, precision, sensitivity, and specificity. From Table 1 it can be seen that polynomial and RBF kernel function performances are similar with accuracy, precision, sensitivity and specificity of $84 \%, 100 \%, 100 \%$ and $100 \%$ respectively. The higher specificity and sensitivity for non-linear SVM clearly indicates that the boundary separating CIN-I and EH samples are not linear. For the calculation of specificity and sensitivity the samples lying on the boundary between the two regions have not been considered. If we only consider the samples lying on either side of the boundary then RBF kernel performs better than others. The RBF kernel based SVM classification results of lifetime and PC scores for training and validation data between EH and CIN-I are shown in Figure 4. In the validation results in Figure $4 \mathrm{~b}, \mathrm{~d}$ two CIN-I samples are lying outside the CIN-I regions for both the cases, while all the normal samples are clearly distinguished. The low specificity can be attributed to lesser number of training samples used. As the number of samples increases, the separating hyperplane will become more robust and will improve the classification accuracy.

Table 1. SVM model results using linear, polynomial and Gaussian radial basis kernel functions for training data set.

\begin{tabular}{lccc}
\hline & Linear & Polynomial Kernel (Order-3) & RBF Kernel \\
\hline Accuracy & 81 & 84 & 84 \\
Precission & 92 & 100 & 100 \\
Sensitivity & 92 & 100 & 100 \\
Specificity & 93 & 100 & 100 \\
\hline
\end{tabular}

Table 2 shows the sensitivity and specificity for lifetime, PCA and their combined results for both training and validation data, respectively. As we have not considered the data lying on boundary for sensitivity and specificity calculation, it becomes $100 \%$ for training data set. For training data both the lifetime and PC scores have similar specificity and sensitivity but in case of validation data PCA performs better than fluorescence lifetime. The combined results obtained from application of SVM shows a better sensitivity as intended. 
Table 2. Comparison of sensitivity and specificity for the results obtained from lifetime, PCA and their combined results.

\begin{tabular}{ccccc}
\hline & Sensitivity Training & Specificity Training & Sensitivity Validation & Specificity Validation \\
\hline Lifetime & 100 & 100 & 60 & 100 \\
PCA & 100 & 100 & 67 & 100 \\
Combined result & 100 & 100 & 87 & 100 \\
\hline
\end{tabular}

\section{Materials and Methods}

\subsection{Sample Collection}

Fresh human cervical tissue samples used in this ex-vivo experiment were obtained from GSVM medical college, Kanpur, Uttar Pradesh, India. The freshly resected tissue samples were stored in ice for transportation to the lab. Before performing the experiment, the samples were thawed to room temperature and then rinsed with saline water to remove superficial blood. The experiment was performed within $4 \mathrm{~h}$ of biopsy. Based on visual examination, samples were labeled as normal and abnormal. After completing the experiment, the samples were sent back to the medical college for histopathology from which the samples were confirmed as EH and CIN-I. EH is declared as normal but its epithelial thickness is almost double compared to normal tissue. Total number of $10 \mathrm{EH}$ samples with 25 sites, 11 CIN-I samples with 20 sites and 1 CIN-II sample with 2 sites have been examined in this study. Out of which 16 sites from each group has been used as training data and 9 sites from EH and 4 sites from CIN-I and 2 sites from CIN-II has been used as validation data.

\subsection{Data Collection}

LaVision ICCD and PicoQuant picosecond pulsed diode laser ( $375 \mathrm{~nm}$ wavelength with pulse width of $48 \mathrm{ps}$, repetition rate $40 \mathrm{MHz} \&$ average power $0.5 \mathrm{~mW}$ ) driven by PDL 800-B driver was used to capture fluorescence lifetime images. The pulsed signal from a single mode fiber was collimated using a achromatic lens (Thorlab $\mathrm{f}=75 \mathrm{~mm}$ ). Fluorescence signal from the tissue was collected using a $450 \mathrm{~nm}$ wavelength long pass filter and imaged onto the ICCD through a camera lens (Nikon AF Nikkor $50 \mathrm{~mm} \mathrm{f} / 1.8 \mathrm{D}$ ). Fluorescence lifetime imaging system comprises a high rate imager (HRI), ICCD and a high rate delay generator (HDG). HRI controls the functioning of image intensifier and HDG provides the desired delay of $100 \mathrm{ps}$ with respect to excitation pulse. The pulses were synchronized to capture FLI from the sample at delay steps of 100 ps with respect to excitation pulse. Control of components and data collection were done by a "Davis" user interface software.

Figure 5 displays the block diagram of experimental setup of fluorescence lifetime imaging system. A $375 \mathrm{~nm}$ pulsed diode laser was used to excite the cervical tissue sample and the FLI were captured with a gate width of $200 \mathrm{ps}$ at steps of $100 \mathrm{ps}$ time delay from the excitation pulse. For good SNR at each pixel the CCD acquisition time was set at $3000 \mathrm{~ms}$. Fluorescence decays were recorded by shifting the time gate in steps of 100 ps over 12.7 nanoseconds. 


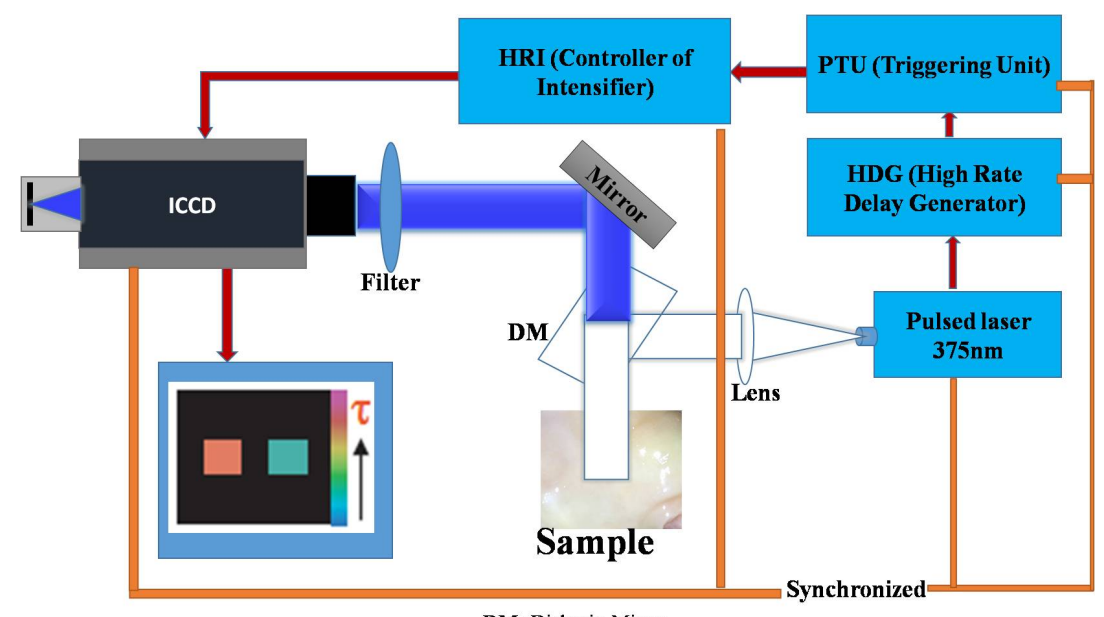

DM: Dichroic Mirror

Figure 5. Block diagram of fluorescence lifetime imaging system for capturing time- resolved fluorescence images.

\subsection{Data Analysis}

\subsubsection{Fluorescence Lifetime}

Fluorescence lifetime imaging: Fluorescence intensity decays at every pixel were fitted to a double exponential (Equation (1)) using a non-linear least square minimization scheme. The fast and slow components of lifetimes for all pixels were then displayed separately as FLI. The code for the fitting is written by using "fit" function available in MATLAB.

Average fluorescence lifetime decays: Average fluorescence decay profile was fitted to a double exponential $\chi^{2}(=0.9999)$. The double exponential function used to fit data is shown in Equation (1). The fitting here has been performed using the curve fitting tool available in MATLAB.

$$
I(t)=\alpha_{1} e^{-\frac{t}{\tau_{1}}}+\alpha_{2} e^{-\frac{t}{\tau_{2}}}
$$

where $\alpha_{1}$ and $\alpha_{2}$ are the amplitudes at $t=0 ; \tau_{1}$ and $\tau_{2}$ are the fluorescence lifetimes.

\subsubsection{Principal Component Analysis (PCA)}

PCA is applied to the average fluorescence decay for the dimensional reduction without loss of any feature. PCA reduces the dimension of the data by finding the orthogonal linear combination (the principal components) of the original variables having largest variance. PCA captures the effect of absorption and scattering through the eigenvectors [29]. To extract principal components of the timeresolved fluorescence signal, a correlation matrix $\mathrm{C}$ is constructed.

$$
C_{i j}=\frac{1}{N} \sum_{i=1}^{N} \delta I_{i}^{T}(k) \delta I_{j}(k)=\frac{A^{T} A}{N}
$$

where, $\delta I_{j}(k)=A_{j k}$ is the mean subtracted intensity divided by the standard deviation, computed over the samples at each time. Index $i$ varies from 1 to 75 , representing fluorescence decay profile and $k$ is the number of samples. The eigenvectors and the eigenvalues of the correlation matrix are extracted using singular value decomposition. The eigenvectors are rearranged in descending order of their eigenvalues. First principal component is the eigenvector corresponding to highest eigenvalue of the matrix, similarly the 2nd PC, 3rd PC, 4th PC are called according to descending order of eigenvalues. PC scores represent the projection of data on the principal components and hence carry useful information, which can be used for classification purposes. The code for this study is written in MATLAB. 


\subsubsection{Support Vector Machine (SVM)}

SVM is a machine learning technique that utilizuses the structural risk minimization (SRM) scheme of statistical machine learning and forms an optical separating hyperplane (OSH) which maximizes the width of the margin between different classes. The OSH minimizes the risk of misclassifying not only the data points in the training set but also yet-to-be-seen data points of the test set for a fixed but unknown probability distribution of the data thereby following the SRM principle. The training data points lying far from the decision boundary receives zero weight while the data points close to the decision boundary have non-zero weight. Support vectors are the training data samples along the hyperplanes near the class boundary and the margin is the distance between support vectors and the class boundary. A classification task involves with training and testing data which consists of some data instances. At each instance, the training set contains one "target value" (class labels) and several "attributes" (features). The two-class decision function defined by an SVM classifier is given by

$$
D(x)=\operatorname{sign}\left[\sum_{\vee x_{i} \in S} \alpha_{i} \lambda_{i} K\left(x_{i}, x_{j}\right)+\alpha_{0}\right]
$$

where $K\left(x_{i}, x_{j}\right)$ is the kernel function of a new data point $x_{j}$ (to be classified) and a set of training data points $x_{i}, S$ is the set of support vectors (a subset of training set), and $\lambda_{i}= \pm 1$ is the label of training data points $x_{i}$ and $\alpha_{i} \geq 0$ are the Lagrange multipliers for OSH. There are three most commonly used kernel functions, which are the linear kernel $K\left(x_{i}, x_{j}\right)=x_{i}^{T} x_{j}$, the polynomial kernel $K\left(x_{i}, x_{j}\right)=\left(x_{i}^{T} x_{j}+1\right)^{d}$ and the Gaussian or RBF kernel $K\left(x_{i}, x_{j}\right)=\exp \left(-\left\|x_{i}-x_{j}\right\|^{2}\right)$. Figure 6 displays the flow chart of the study.

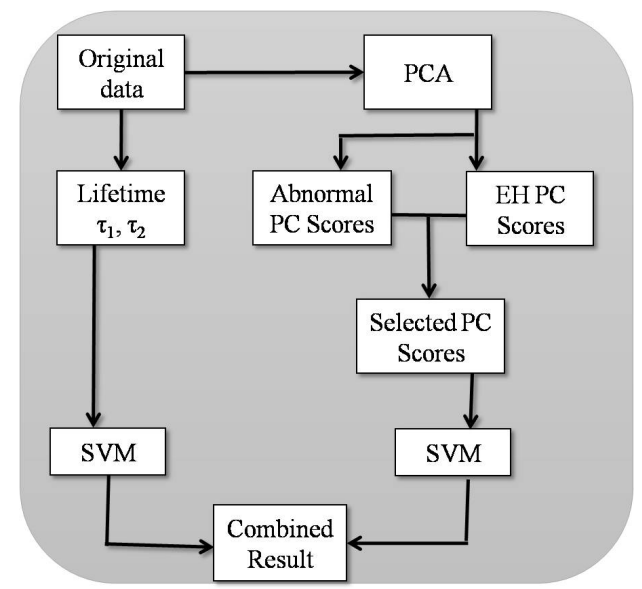

Figure 6. Flow chart of the algorithm used for analysis, PCA: Principal Component Analysis, SVM: Support Vector Machine.

\section{Conclusions}

Fluorescence lifetime distinguishes EH and CIN-I tissues and the distinction improves by the application of PCA. The difference is attributed to the variation in scattering between EH and CIN-I tissues. Application of PCA enhances the discrimination as the principal components capture both absorption and scattering effects [29]. Further application of SVM on fluorescence lifetime and PC scores quantifies the distinction with even better accuracy. Finally, the combined results of both fluorescence lifetime and PCA significantly improves the sensitivity and hence the diagnostic capability. The preliminary study suggests that the fluorescence lifetime and PCA combined with RBF kernel function in SVM has the potential to demarcate abnormal from EH samples and performance of this method will become more robust with a larger data set.

Author Contributions: G.R.S. did all the experiments, analysis and wrote the paper. P.S. helped in performing the experiments. A.P. provided guidance throughout the experimental and analytical work giving useful advice 
to remove any bottleneck during the work and critically examined the manuscript. K.P. provided the biopsied cervical tissue samples, while C.K. took care of all the histopathology work.

Funding: The equipment was funded by a project from Department of Biotechnology, Ministry of Science and Technology, India, grant number BT/PR4497/MED/30/706/2012 and Indian Institute of Technology Kanpur, India.

Acknowledgments: Preliminary data were part of the project funded by Department of Biotechnology, Ministry of Science and Technology, India and Indian Institute of Technology Kanpur, India. Gyana Ranjan Sahoo would like to thank Prabodh Kumar Pandey for technical help.

Conflicts of Interest: The authors declare no conflict of interest.

\section{References}

1. Alfano, R.R.; Tata, D.B.; Cordero, J.; Tomashefsky, P.; Longo, F.; Alfano, M. Laser induced fluorescence spectroscopy from native cancerous and normal tissue. IEEE J. Quantum Electron. 1984, 20, 1507-1511. [CrossRef]

2. Alfano, R.R.; Tang, G.C.; Pradhan, A.; Lam, W.; Choy, D.; Opher, E. Fluorescence spectra from cancerous and normal human breast and lung tissues. IEEE J. Quantum Electron. 1987, 23, 1806-1811. [CrossRef]

3. Alfano, R.R.; Pradhan, A.; Tang, G.C.; Wahl, S.J. Optical spectroscopic diagnosis of cancer and normal breast tissues. JOSA B 1989, 6, 1015-1023. [CrossRef]

4. Pu, Y.; Wang, W.; Yang, Y.; Alfano, R.R. Stokes shift spectroscopy highlights differences of cancerous and normal human tissues. Opt. Lett. 2012, 37, 3360-3362. [CrossRef] [PubMed]

5. Heintzelman, D.L.; Utzinger, U.; Fuchs, H.; Zuluaga, A.; Gossage, K.; Gillenwater, A.M.; Jacob, R.; Kemp, B.; Richards-Kortum, R.R. Optimal Excitation Wavelengths for In Vivo Detection of Oral Neoplasia Using Fluorescence Spectroscopy. Photochem. Photobiol. 2000, 72, 103-113. [CrossRef]

6. Brookner, C.K.; Utzinger, U.; Staerkel, G.; Richards-Kortum, R.R.; Mitchell, M.F. Cervical fluorescence of normal women. Lasers Surg. Med. 1999, 24, 29-37. [CrossRef]

7. Grossman, N.; Ilovitz, E.; Chaims, O.; Salman, A.; Jagannathan, R.; Mark, S.; Cohen, B.; Gopas, J.; Mordechai, S. Fluorescence spectroscopy for detection of malignancy: H-ras overexpressing fibroblasts as a model. J. Biochem. Biophys. Methods 2001, 50, 53-63. [CrossRef]

8. Pu, Y.; Wang, W.; Yang, Y.; Alfano, R.R. Stokes shift spectroscopic analysis of multifluorophores for human cancer detection in breast and prostate tissues. J. Biomed. Opt. 2013, 18, 017005. [CrossRef]

9. Ramanujam, N.; Mitchell, M.F.; Mahadevan, A.; Warren, S.; Thomsen, S.; Silva, E.; Kortum, R.R. In vivo diagnosis of cervical intraepithelial neoplasia using 337-nm-excited laser-induced fluorescence. Proc. Natl. Acad. Sci. USA 1994, 91, 10193-10197. [CrossRef]

10. Chidananda, S.M.; Satyamoorthy, K.; Rai, L.; Manjunath, A.P.; Kartha, V.B. Optical diagnosis of cervical cancer by fluorescence spectroscopy technique. Int. J. Cancer 2006, 119, 139-145. [CrossRef]

11. Chang, S.K.; Mirabal, Y.N.; Atkinson, E.N.; Cox, D.D.; Malpica, A.; Mitchell, M.F.; Kortum, R.R. Combined reflectance and fluorescence spectroscopy for in vivo detection of cervical pre-cancer. J. Biomed. Opt. 2005, 10, 024031. [CrossRef] [PubMed]

12. Fang, Q.; Papaioannou, T.; Jo, J.A.; Vaitha, R.; Shastry, K.; Marcu, L. Time-domain laser-induced fluorescence spectroscopy apparatus for clinical diagnostics. Rev. Sci. Instrum. 2004, 75, 151-162. [CrossRef]

13. Pitts, J.D.; Mycek, M.A. Design and development of a rapid acquisition laser-based fluorometer with simultaneous spectral and temporal resolution. Rev. Sci. Instrum. 2001, 72, 3061-3072. [CrossRef]

14. Mycek, M.A.; Schomacker, K.T.; Nishioka, N.S. Colonic polyp differentiation using time-resolved autofluorescence spectroscopy. Gastrointest. Endosc. 1998, 48, 390-394. [CrossRef]

15. Elson, D.; Requejo-Isidro, J.; Munro, I.; Reavell, F.; Siegel, J.; Suhling, K.; Tadrous, P.; Benninger, R.; Lanigan, P.; McGinty, J.; et al. Time-domain fluorescence lifetime imaging applied to biological tissue. Photochem. Photobiol. Sci. 2004, 3, 795-801. [CrossRef] [PubMed]

16. Vishwanath, K.; Pogue, B.; Mycek, M.A. Quantitative fluorescence lifetime spectroscopy in turbid media: Comparison of theoretical, experimental and computational methods. Phys. Med. Biol. 2002 47, 3387. [CrossRef] 
17. Pradhan, A.; Das, B.B.; Yoo, K.M.; Cleary, J.; Prudente, R.; Celmer, E.; Alfano, R.R. Time-resolved UV photoexcited fluorescence kinetics from malignant and non-malignant human breast tissues. Lasers Life Sci. 1992, 4, 225-234.

18. Tata, D.B.; Foresti, M.; Cordero, J.; Tomashefsky, P.; Alfano, M.A.; Alfano, R.R. Fluorescence polarization spectroscopy and time-resolved fluorescence kinetics of native cancerous and normal rat kidney tissues. Biophys. J. 1986, 50, 463-469. . [CrossRef]

19. Tadrous, P.J.; Siegel, J.; French, P.M.; Shousha, S.; Lalani EN Stamp, G.W. Fluorescence lifetime imaging of unstained tissues: Early results in human breast cancer. J. Pathol. 2003, 199, 309-317. [CrossRef]

20. Das, B.; Shi, L.; Budansky, Y.; Rodriguez-Contreras, A.; Alfano, R. Alzheimer mouse brain tissue measured by time resolved fluorescence spectroscopy using single-and multi-photon excitation of label free native molecules. J. Biophotonics 2018, 11, 201600318. [CrossRef]

21. Sun, Y.; Phipps, J.; Elson, D.S.; Stoy, H.; Tinling, S.; Meier, J.; Poirier, B.; Chuang, F.S.; Farwell, D.G.; Marcu, L. Fluorescence lifetime imaging microscopy: In vivo application to diagnosis of oral carcinoma. Opt. Lett. 2009, 34, 2081-2083. [CrossRef] [PubMed]

22. Kalaivani, R.; Masilamani, V.; AlSalhi, M.S.; Devanesan, S.; Ramamurthy, P.; Palled, S.R.; Ganesh, K.M. Cervical cancer detection by time-resolved spectra of blood components. J. Biomed. Opt. 2014, 19, 057011. [CrossRef]

23. Gu, J.; Fu, C.Y.; Ng, B.K.; Liu, L.B.; Lim-Tan, S.K.; Lee, C.G.L. Enhancement of early cervical cancer diagnosis with epithelial layer analysis of fluorescence lifetime images. PLoS ONE 2015, 10, 0125706. [CrossRef] [PubMed]

24. Evan Gerard, I.; Karen, H.V. Proliferation, cell cycle and apoptosis in cancer. Nature 2001, 411, 342. [CrossRef] [PubMed]

25. Drezek, R.A.; Guillaud, M.; Collier, T.G.; Boiko, I.; Malpica, A.; MacAulay, C.E.; Richards-Kortum, R.R. Light scattering from cervical cells throughout neoplastic progression: Influence of nuclear morphology, DNA content, and chromatin texture. J. Biomed. Opt. 2003, 8, 7-17. [CrossRef] [PubMed]

26. Devi, S.; Panigrahi, P.K.; Pradhan, A. Detecting cervical cancer progression through extracted intrinsic fluorescence and principal component analysis. J. Biomed. Opt. 2014, 19, 127003. [CrossRef] [PubMed]

27. Georgakoudi, I.; Jacobson, B.C.; Müller, M.G.; Sheets, E.E.; Badizadegan, K.; Carr-Locke, D.L.; Crum, C.P.; Boone, C.W.; Dasari, R.R.; Van Dam, J.; et al. NAD (P) H and collagen as in vivo quantitative fluorescent biomarkers of epithelial precancerous changes. Cancer Res. 2002, 62, 682-687.

28. Alfano, R.R. Advances in ultrafast time resolved fluorescence physics for cancer detection in optical biopsy. AIP Adv. 2012, 2, 011103. [CrossRef]

29. Singh, P.; Sahoo, G.R.; Pradhan, A. Spatio-temporal map for early cancer detection: Proof of concept. J. Biophotonics 2018. [CrossRef]

30. Drezek, R.A.; Sokolov, K.V.; Utzinger, U.; Boiko, I.; Malpica, A.; Mitchell, M.F.; Richards-Kortum, R.R. Understanding the contributions of NADH and collagen to cervical tissue fluorescence spectra: Modeling, measurements, and implications. J. Biomed. Opt. 2001, 6, 385-397. [CrossRef]

31. Kamath, S.D.; Bhat, R.A.; Ray, S.; Mahato, K.K. Autofluorescence of normal, benign, and malignant ovarian tissues: A pilot study. Photomed. Laser Surg. 2009, 27, 325-335. [CrossRef] [PubMed]

32. Seo, J.; An, Y.; Lee, J.; Ku, T.; Kang, Y.; Ahn, C.W.; Choi, C. Principal component analysis of dynamic fluorescence images for diagnosis of diabetic vasculopathy. J. Biomed. Opt. 2016, 21, 046003. [CrossRef]

33. Pu, H.; He, W.; Zhang, G.; Zhang, B.; Liu, F.; Zhang, Y.; Luo, J.; Bai, J. Separating structures of different fluorophore concentrations by principal component analysis on multispectral excitation-resolved fluorescence tomography images. Biomed. Opt. Express 2013, 4, 1829-1845. [CrossRef] [PubMed]

34. Hillman, E.M.; Moore, A. All-optical anatomical co-registration for molecular imaging of small animals using dynamic contrast. Nat. Photonics 2007, 1, 526. [CrossRef]

35. Welshe, K.; Sherlock, S.P.; Dai, H. Deep-tissue anatomical imaging of mice using carbon nanotube fluorophores in the second near-infrared window. Appl. Phys. Sci. 2011, 108, 8943-8948. [CrossRef]

36. Liu, X.; Wang, D.; Liu, F.; Bai, J. Principal component analysis of dynamic fluorescence diffuse optical tomography images. Opt. Express 2010, 18, 6300-6314. [CrossRef]

37. Cortes, C.; Vladimir, V. Support-vector networks. Mach. Learn. 1995, 20, 273-297. [CrossRef]

38. Chapelle, O.; Vapnik, V.; Bengio, Y. Model selection for small sample regression. Mach. Learn. 2002, 48, 9-23.:1013943418833. [CrossRef] 
39. Liu, Y.; Zheng, Y.F. FS SFS A novel feature selection method for support vector machines. Common. Comput. Inf. Sci. 2006, 39, 1333-1345. [CrossRef]

40. Acir, N. A support vector machine classifier algorithm based on a perturbation method and its application to ECG beat recognition systems. Expert Syst. Appl. 2006, 31, 150-158. [CrossRef]

41. Rejani, Y.; Selvi, S.T. Early detection of breast cancer using SVM classifier technique. Int. J. Comput. Sci. Eng. 2009, 1, 127-130.

42. Majumder, S.K.; Ghosh, N.; Gupta, P.K. Support vector machine for optical diagnosis of cancer. J. Biomed. Opt. 2005, 10, 024034. [CrossRef] [PubMed]

43. Mukhopadhyay, S.; Kurmi, I.; Dey, R.; Das, N.K.; Pradhan, S.; Pradhan, A.; Ghosh, N.; Panigrahi, P.K.; Mohanty, S. Optical diagnosis of colon and cervical cancer by support vector machine. In Biophotonics: Photonic Solutions for Better Health Care V; SPIE Photonics Europe: Brussels, Belgium, 2016; Volume 9887.

44. Girosi, F.; Jones, M.; Poggio, T. Regularization theory and neural networks architectures. Neural Comput. 1995, 7, 219-269. . [CrossRef]

45. Smola, A.J.; Scholkopf, B.; Muller, K.R. The connection between regularization operators and support vector kernels. Neural Netw. 1998, 11, 637-649. [CrossRef]

46. Lee, K.C.; Siegel, J.; Webb, S.E.D.; Leveque-Fort, S.; Cole, M.J.; Jones, R.; Dowling, K.; Lever, M.J.; French, P.M.W. Application of the stretched exponential function to fluorescence lifetime imaging. Biophys. J. 2001, 81, 1265-1274. [CrossRef]

(C) 2018 by the authors. Licensee MDPI, Basel, Switzerland. This article is an open access article distributed under the terms and conditions of the Creative Commons Attribution (CC BY) license (http:/ / creativecommons.org/licenses/by/4.0/). 